Pour abréger, on peut appeler stèle (1) un cylindre central, et qualifier de monostélique la première de ces dispositions, de polystélique la seconde et d'astélique la troisième. A son tour, la structure polystélique peut être dite dialystèle, lorsque les cylindres centraux sont isolés (Auricula ursi, etc.); gamostèle, lorsqu'ils sont fusionnés en anneau (A. japonica, etc.), Les mêmes termes s'appliquent également bien aux feuilles, dont le limbe est toujours astélique, mais dont le pétiole est, suivant les cas, monostẻlique (Solanées, Cucurbitacées, etc.), polystélique (beaucoup de Fougères, Gunnera, etc.) ou astélique (Composées, etc.).

Quant à la racine, elle est monostélique dans la presque totalité des cas. Chez les Lycopodiacées seules, son cylindre central se bifurque comme celui de la tige, et l'on peut dire qu'elle est polystélique quand on la considère dans sa totalité.

En résumé, on peut ranger les différents modes de groupement des faisceaux libériens, ligneux ou libéro-ligneux, de la manière suivante:

$1^{\circ}$ Structure monostélique. - Toutes les racines, à l'exception de celles des Lycopodiacées, la plupart des tiges de Phanérogames, le pétiole des Solanées, des Cueurbitacées, etc.

$2^{\circ}$ Structure polystélique. - La tige des Auricules, des Gunnera, de la plupart des Fougères, des Marsiliacées, des Sélaginelles, des Lycopodes, etc.; le pétiole de beaucoup de Fougères; la racine des Lycopodiacées.

$3^{\circ}$ Structure astélique. - La tige des Nymphéacées, de diverses Renoncules, de l'Hydrocleis; le limbe des feuilles.

M. Duchartre propose de supprimer, pour être logique, le mot cylindre central.

M. Douliot répond qu'il ne voit aucun inconvénient à nommer stèle le cylindre central unique d'une tige normale.

M. Van Tieghem fait à la Société la communication suivante :

\title{
INVERSION DU SUCRE DE CANNE PAR LE POLLEN,
} par M. Ph. vav THEGuEn.

J'ai montré, il y a déjá quinze ans, que le grain de pollen germe et développe son tube pollinique dans un milieu de culture appropriẻ, renfermant un aliment ternaire qui peut être du sucre de Canne (2). Pendant cette germination et ce développement, le sucre de Canne est-il

(1) De $\sigma \cdot \eta \lambda \lambda$, colonne

(2) $\mathrm{Ph}$. Van Tieghem, Recherches physiologiques sur la végétation libre du pollen et e l'ovule (Ann. des sc. nat. Bor. $5^{\circ}$ série, XII, 1872). 
absorbé comme tel, ou subit-il de la part du grain ou du tube une inversion préalable, pour ne s'introduire dans la plantule qu'à l'état de glucose et de lévulose? Si cette inversion a lieu, l'invertine qui la produit se forme-t-elle seulement au cours de la germination et du développement du tube pollinique, ou préexiste-t-elle dans le grain de pollen mûr? Ce sont les deux questions que je me suis proposé de résoudre.

Dans les premiers essais dont je rends comple aujourd'hui à la Société, je me suis servi du pollen de Safran (Crocus vernus), de Jacinthe (Hyacinthus orientalis), de Narcisse (Narcissus odorus et N. Pseudonarcissus), de Giroflée (Cheiranthus Cheiri) et de Violette (Viola odorata). Ces pollens sont dépourvus d'amidon; ils jaunissent tout entiers par l'iode. Ils ne réduisent pas la liqueur cupropotassique, et par conséquent ne contiennent pas de glucose; ils ne la réduisent pas davantage après l'ébullition avec l'acide sulfurique étendu, et par suite ne renferment ni dextrine, ni sucre de Canne. Par l'absence de glucose, ils ressemblent à ceux du Noisetier et du Pin, analysés avec tant de soin par M. de Planta (1); mais ils en diffèrent par l'absence d'amidon et du sucre de Canne. Ce chimiste, en effet, a trouvé, dans le pollen du Noisetier 5 pour 100 d'amidon et 14 pour 100 de sucre de Canne, dans le pollen du Pin 7 pour 100 d'amidon et 11 pour 100 de sucre de Canne.

Dans un verre de montre contenant $5^{\text {ce }}$ d'une dissolution de sucre de Canne à 10 pour 100 , on sème une petite quantité de l'un quelconque de ces pollens, notamment de ceux de Safran et de Narcisse, sur lesquels les ecsais ont été le plus fréquemment répétés. Les précautions sont prises pour éviter le développement des Champignons et des Bactéries dans la liqueur. Après vingt-quatre heures, le liquide filtré réduit fortement le liquide cupropotassique, ce qui prouve qu'une notable proportion du sucre de Canne a étẻ transformée en sucre inverti. On s'assure en même temps, par l'examen microscopique, que bon nombre de grains ont commencé à germer et à pousser leur tube.

Pour savoir si l'invertine qui a agi dans cette première série d'expériences préexiste dans le pollen mûr, ou si elle ne s'y forme que pendant sa germination, on a fait une seconde série d'essais. Le liquide de culture est additionné de quelques gouttes de chloroforme et enfermé dans de petits flacons à demi remplis qu'on bouche après l'introduction du pollen. Le chloroforme interdit toute germination des grains de pollen, comme aussi tout développement d'organismes étrangers; mais il n'empêche pas l'invertine d'agir sur le sucre de Canne. Après vingt-quatre heures, le liquide est filtré, débarrassé du chloroforme, et traité par la liqueur cupropotassique, qu'il réduit fortement. La réduction est plus forte après

(1) Landwirthschaftl. Versuchsstationen, 60 série, 1884, p. 97, et 1885, p. 216. 
quarante-huit heures et progresse encore, mais plus faiblement, les jours suivants.

Citons quelques chiffres. Le $1^{\text {er }}$ avril, des tubes contenant chacun $66^{\mathrm{cc}}$ de solution sucrée renfermant 0 sr, 6 de sucre de Canne et quelques gouttes de chloroforme sont additionnés d'une quantité sensiblement égale de pollen de Safran et mis bouchés à l'étuve à 35 degrés. Le 2 avril, après vingt-quatre heures de séjour à l'étuve, le liquide d'un des tubes contient $0 \mathrm{gr}, 2$ de sucre inverti; un tiers de sucre de Canne a donc étẻ transformé. Le 7 avril, après cinq jours, le liquide d'un second tube renferme $0{ }^{g r}, 4$ de sucre inverti ; les deux tiers du sucre de Canne ont subi la transformation. Avec le pollen du Narcisse faux-Narcisse, on a obtenu dans les mêmes conditions un résultat analogue, savoir, inversion d'environ un tiers du sucre de Canne après vingt-quatre heures, d'environ deux tiers aṕrès cinq jours.

L'invertine existe done toute formée dans les grains de pollen mûrs. Elle s'y trouve en proportion relativement considérable, si l'on en juge par la petite quantité de pollen introduit et par la forte proportion de glucose formé.

Pour ne pas s'étonner de voir des grains de pollen dépourvus de sucre de Canne produire ainsi de l'invertine, qui parait devoir leur être inutile, il suffit de se rappeler que les exemples de ce genre sont très fréquents. Ainsi, dans la cuve du brasseur, la levûre de bière fait de l'invertine, qui est pour elle sans emploi, puisque le moùt de bière ne renferme pas de sucre de Canne; de même, le Penicillium glauque et d'autres moisissures font de l'invertine quand on les cultive dans un liquide sucré avec du glucose. Les pollens étudiés dans ce travail ne font done qu'apporter une preuve de plus à l'appui de ce fait, que la cellule vivante capable de faire de l'invertine la produit par l'effet même de sa nutrition passée, et indépendamment de l'emploi de cette substance pour sa nutrition à venir.

Quelques essais comparatifs avec des spores de Lycopode et de quelques Fougères ont donné des résultats analogues à ceux qui viennent d'être signalés pour le pollen. Seulement, l'action inversive de ces spores a paru, toutes choses égales d'ailleurs, beaucoup plus faible que celle du pollen. 


\section{$2 \mathrm{BHL}$ Biodiversity Heritage Library}

Van Tieghem, Phillippe Édouard Léon. 1886. "Inversion Du Sucre De Canne Par Le Pollen." Bulletin de la Société botanique de France 33, 216-218. https://doi.org/10.1080/00378941.1886.10828431.

View This Item Online: https://www.biodiversitylibrary.org/item/8655

DOI: https://doi.org/10.1080/00378941.1886.10828431

Permalink: https://www.biodiversitylibrary.org/partpdf/159004

\section{Holding Institution}

Missouri Botanical Garden, Peter H. Raven Library

\section{Sponsored by}

Missouri Botanical Garden

\section{Copyright \& Reuse}

Copyright Status: Public domain. The BHL considers that this work is no longer under copyright protection.

This document was created from content at the Biodiversity Heritage Library, the world's largest open access digital library for biodiversity literature and archives. Visit BHL at https://www.biodiversitylibrary.org. 